\title{
Oseltamivir once or twice daily safely prevented influenza
}

Hayden FG, Atmar RL, Schilling M, et al, and the Oseltamivir Study Group. Use of the selective oral neuraminidase inhibitor oseltamivir to prevent influenza. N Engl J Med 1999 Oct 28;341:1336-43.

\section{QUESTION: Is oral oseltamivir safe and effective in preventing naturally occurring influenza virus infection?}

\section{Design}

Two 6 week randomised (allocation concealed*), blinded (clinicians and patients),* placebo controlled trials during influenza season.

\section{Setting}

3 centres in Virginia, 2 in Texas, and 1 in Kansas, USA.

\section{Participants}

1562 people who were 18 to 65 years of age (mean age $35 \mathrm{y}, 63 \%$ women) and were recruited by advertisement. Exclusion criteria were influenza vaccination in the previous year, meeting $\geqslant 1$ criterion for influenza immunisation according to current (US) guidelines, acute respiratory illness with fever in the previous week, pregnancy, or potential for pregnancy. 1559 participants took $\geqslant 1$ dose of the assigned study medication and were included in the analysis (intention to treat).

\section{Intervention}

Participants were allocated to oral oseltamivir, $75 \mathrm{mg}$ once $(n=520)$ or twice $(n=520)$ daily, or to placebo $(n=519)$ for 6 weeks, beginning when influenza virus activity increased at the local study sites.

\section{Main outcome measures}

Laboratory confirmed influenza-like illness (laboratory confirmation was culture of influenza virus within $2 \mathrm{~d}$ of the onset of influenza symptoms or antibody titer on haemagglutination inhibition testing $\geqslant 4$ times the baseline titer or both). Adverse events were also assessed.

\section{Main results}

Fewer patients receiving oseltamivir developed laboratory confirmed influenza than did patients receiving placebo (once daily $\mathrm{p}<0.001$, twice daily $\mathrm{p}=0.001$ ) (table). Rates of influenza were higher at the 3 Virginia sites than at the Texas and Kansas sites. Oseltamivir reduced influenza in Virginia (protective efficacy $82 \%$, 95\% CI $60 \%$ to $93 \%$; $\mathrm{p}<0.001)$ but showed no significant reduction in Texas and Kansas (protective efficacy $50 \%$, CI $-23 \%$ to $93 \%$; $\mathrm{p}=0.39$ ). Nausea and vomiting were reported by more oseltamivir recipients than placebo recipients but did not contribute to discontinuation of treatment (table).

\section{Conclusion}

Oseltamivir once or twice daily was effective and safe in preventing influenza.

*See glossary.

Oseltamivir, $75 \mathrm{mg}$ once (Osel qd) or twice (Osel bid) daily,v placebo for preventing influenzat

\begin{tabular}{|c|c|c|c|c|}
\hline Outcomes at 6 wk & Comparison & Event rates & RRR (95\% Cl) & NNT (Cl) \\
\hline \multirow{2}{*}{$\begin{array}{l}\text { Laboratory confirmed } \\
\text { influenza-like illness }\end{array}$} & Osel bid $v$ placebo & $1.3 \% \vee 4.8 \%$ & $72 \%(40$ to 89$)$ & 29 (17 to 69$)$ \\
\hline & & & RRI (Cl) & NNH (Cl) \\
\hline Nausea & Osel qd $v$ placebo & $12.1 \% \vee 7.1 \%$ & $70 \%(16$ to 150$)$ & 20 (12 to 70$)$ \\
\hline \multirow[t]{2}{*}{ Vomiting } & Osel qd $v$ placebo & $2.5 \% \vee 0.8 \%$ & $224 \%$ (12 to 840$)$ & 58 (28 to 479$)$ \\
\hline & Osel bid $v$ placebo & $2.7 \% \vee 0.8 \%$ & $249 \%$ (22 to 904$)$ & 52 (26 to 267 ) \\
\hline
\end{tabular}

Source of funding: Hoffmann-LaRoche.

For correspondence: Dr FG Hayden, Department of Medicine, Box 473, University of Virginia Health Sciences Center, Charlottesville, VA 22908, USA. Fax +1 8049249065

\section{COMMENTARY_continued from previous page}

within 2 weeks of completing the 6 week prophylaxis period, including 5 participants who had received oseltamivir. The neuraminidase inhibitors may protect against illness only for as long as the medication is actually taken. More data on the effectiveness of the neuraminidase inhibitors for the prevention of influenza among elderly people, nursing home residents, and other high risk groups are needed.

How should the neuraminidase inhibitors be used? Immunisation remains the mainstay of efforts for the prevention and control of influenza. However, antiviral agents, including the neuraminidase inhibitors, are important adjuncts to vaccination for prevention and treatment during interpandemic periods for people who have not been vaccinated, who develop influenza even if they have been vaccinated, and who travel between May and September to areas of the world with influenza activity. Decisions about which antiviral agent to use should be based on the relative importance of spectrum of activity, side effects, risk for emergence of resistance, and cost. Given the need to start treatment within 2 days of the onset of symptoms, providers will have to develop efficient processes for accurate diagnosis and timely prescribing of these agents if they are to be used widely. The antirivals will also play an important role during the next pandemic, providing critical protection during times when sufficient supplies of vaccine may not be available.

Kristin L Nichol, MD, MPH Veterans Affairs Medical Center Minneapolis, Minnesota, USA

1 MMWR Morb Mortal Wkly Rep. 1999;48(RR14):1-9.

2 Hayden FG, Osterhaus AD, Treanor IJ, et al. N Engl J Med 1997;337:874-80.

3 The MIST (Management of Influenza in the Southern Hemisphere Trialists) Study Group. Lancet 1998;352:1877-81.

4 Treanor JJ, Hayden FG, Vrooman PS, et al.JAMA 2000;283:1016-24.

5 Monto AS, Robinson DP, Herlocher ML, et al.JAMA 1999;282:31-5. 